PROCEEDINGS OF THE

AMERICAN MATHEMATICAL SOCIETY

Volume 130, Number 10, Pages 2947-2958

S 0002-9939(02)06405-5

Article electronically published on April 22, 2002

\title{
POSITIVE SOLUTIONS OF A LOGISTIC EQUATION ON UNBOUNDED INTERVALS
}

\author{
LI MA AND XINGWANG XU
}

(Communicated by Carmen C. Chicone)

\begin{abstract}
In this paper, we study the existence of positive solutions of a oneparameter family of logistic equations on $R_{+}$or on $R$. These equations are stationary versions of the Fisher equations and the KPP equations. We also study the blow-up region of a sequence of the solutions when the parameter approaches a critical value and the non-existence of positive solutions beyond the critical value. We use the direct method and the sub and super solution method.
\end{abstract}

\section{INTRODUCTION}

As pointed out in the book written by Buttazzo, Giaguinta, and Hildebrant [1], one-dimensional variational problems deserve special attention. In fact, these kind of problems have their own characters. Sometimes, higher-dimensional variational problems can be reduced to one-dimensional problems.

In this work, we study the existence of positive solutions of the following oneparameter family of logistic equations

$$
u^{\prime \prime}+a f(x) u-b(x) u^{p}=0 \quad \text { in } \quad I,
$$

where $I=(0, \infty)$ is the half real line $R_{+}$or the whole real line $R, p>1, a \in R$ is the parameter, $f(x)$ is a given $C^{1}$ function on $I$, which is positive somewhere, and $b(x)$ is a given non-negative smooth function on $I$. These equations are of interest in mathematical biology. Our equations appear also in Ginzburg-Landau theory of super-conductivity [21]. Such equations were also known as the stationary version of the Fisher equation and KPP equation [20], and they were considered by Kazdan-Warner [6], Ouyang [7], and de Pino [8] in compact manifolds of higher dimensions and Du and Huang [10] in compact domains in the euclidean space $R^{n}$. Remarkably, Ouyang [7] proved a conjecture posed by Kazdan and Warner in [6]. Such problems have also been considered by Li-Tam-Yang [12], Afrouzi-Brown [20] and Du-Ma [18]-[19] on $R^{n}$. For more background material, see [20]. However, to the authors best knowledge, there are very few results on $(1)_{a}$ on $R_{+}$or on $R$.

Received by the editors October 9, 2000 and, in revised form, May 3, 2001.

1991 Mathematics Subject Classification. Primary 34B09, 35J65.

Key words and phrases. Direct method, blow-up, positive solution.

The work of the first author was partially supported by the 973 project of China, a grant from the Ministry of Education, and a scientific grant of Tsinghua University at Beijing. The authors thank the referee for helpful corrections.

(C)2002 American Mathematical Society 
These problems have a close relation with the principal eigenvalue problem

$$
u^{\prime \prime}+\lambda f(x) u=0 \text { in } R .
$$

It is proved by Brown, Cosner, and Fleckinger 4] that if $\int_{R} f(x) d x>0$, then there does not exist a positive principal eigenvalue of (2). This result is also true in dimension two. For more results about principal eigenvalue problems, we refer to the articles [5], 3] and [2]. So we may expect that the study of $(1)_{a}$ has some special properties in dimensions one and two. We will study logistic equations in dimension two in the near future.

For simplicity, we denote the left side of $(1)_{a}$ by the operator $T_{a}(u)$, and we state the results in the case where $I=R_{+}$. Since we will use the sub and super method to study $(1)_{a}$ in the case when $I=R$, the result will be stated later in this article. We now assume that $I=R_{+}$, and we say that $f(x)$ is in class $P$ if $f(x)$ is positive somewhere and satisfies the condition that

$$
\max \{f(x), 0\} \leq p(x), \quad \text { on } \quad R_{+},
$$

where $p(x)$ is some positive function on $R_{+}$with

$$
\int_{R_{+}} x p(x) d x<\infty
$$

With this assumption we can obtain a compactness result, which is of the type of Rellich-Kondrakov imbedding for Sobolev spaces.

We define the zero set

$$
M_{0}=\left\{x \in R_{+} ; b(x)=0\right\},
$$

which will play an important role in the existence problem and the blow-up problem (see [6]).

Assume that $M_{0}$ is a bounded open set in $R$. Let $\lambda_{*}:=\lambda_{1}\left(M_{0}\right)$ and let $\lambda_{1}$ denote the principal eigenvalues of the operator $-u^{\prime \prime}$ with respect to the weighted quotient

$$
Q(u, M):=\int_{M}\left|u^{\prime}\right|^{2} / \int_{M} f(x) u^{2}
$$

where we assume $\int_{M} f(x) u^{2}>0 ; M=M_{0}$ and $M=R_{+}$respectively. We write $\lambda_{*}=+\infty$ when $M_{0}$ is empty. Note that $\lambda_{*} \geq \lambda_{1}$.

We assume that $f$ is positive somewhere outside $M_{0}$. So we have $\lambda_{*}>\lambda_{1}$. Then we will use the direct method to prove the existence of a positive solution of the equation $(1)_{a}$ when $a \in\left(\lambda_{1}, \lambda_{*}\right)$. The idea goes as follows. Define the space $E$ as the completion of $C_{0}^{1}\left(R_{+}\right)$with respect to the norm $\int_{R}\left|u^{\prime}\right|^{2}$. Define the functional

$$
J(u)=\frac{1}{2} \int_{R_{+}}\left(\left|u^{\prime}\right|^{2}-a f(x) u^{2}\right)+\frac{1}{p+1} \int_{R_{+}} b(x)|u|^{p+1}
$$

on $E . J(u)=+\infty$ if $\int_{R_{+}} b(x)|u|^{p+1}-a f(x) u^{2}=+\infty$. Note that by our inequality (*) to be proved in the next section, the functional $J$ is well-defined on $E$. It is easy to see that $J$ is weakly lower semi-continuous on $E$. Assume $a \in\left(\lambda_{1}, \lambda_{*}\right)$. We then prove that the functional $J$ is coercive on $E$ and we get a minimizer of the functional $J$ on $E$. We will show that this minimizer is a non-trivial positive solution of $(1)_{a}$.

We also study the blow-up region of a sequence of the solutions when the parameter approaches the critical value $\lambda_{*}$ and the non-existence of positive solutions beyond the critical value. We use a variational method and a barrier method. 
To state our result, we introduce some notations and concepts. We will write $\|u\|$ as the norm of $u \in H$ and $\|u\|_{q}$ as the norm of $u \in L^{q}\left(R_{+}\right)$where $q>1$. We will write

$$
L^{2}\left(R_{+}, p\right)=L^{2}\left(R_{+}, p d x\right) .
$$

We will sometimes write $M:=R_{+}$.

Our main result is

Theorem 1. Given a positive bounded function $f(x)$ of class $P$ and a non-trivial non-negative smooth function $b$ on $R_{+}$, assume that $f$ is positive somewhere outside the zero set $M_{0}$ of the function $b$. Then the following three assertions are true:

(i) When $a \in\left(\lambda_{1}, \lambda_{*}\right)$, there is a unique positive smooth solution $u_{a} \in H$ of $(1)_{a}$.

(ii) Assume $\lambda_{*}<+\infty$. When $a \rightarrow \lambda_{*}^{-}$, we have that $u_{a} \rightarrow+\infty$ on any compact set $K \subset \subset M_{0}$.

(iii) Assume $\lambda_{*}<+\infty$. When $a \geq \lambda_{*}$, no positive E-solutions of $(1)_{a}$ exist.

We remark that we can extend the existence part of the result above to the following system:

$$
W_{j}^{\prime \prime}+a F_{j}(x) W_{j}-|W|^{p-1} W_{j}=0 \quad \text { on } \quad R_{+},
$$

with $W_{j}(0)=0$ for $j=1, \ldots, N$. Here we write $W=\left(W_{1}, \ldots, W_{N}\right)$ and assume that all the coefficients $F_{j}(x)$ are dominated by functions in class $(P)$. Because the formulation of the result is straightforward, we omit it.

The rest of this paper is organized as follows. In section two, we set up some inequalities and we prove a compactness result. We also discuss the existence of the principal eigenvalue and eigen-function problem (2) on $R_{+}$. Uniqueness and existence of positive $E$-solutions for $(1)_{a}$ will be proved in section three. In section four and in section five, blow-up behavior of a solution sequence and non-existence of positive solutions will be presented. In the final section, we study the existence and uniqueness of positive solutions of $(1)_{a}$ in the case when $I=R$.

\section{Compactness lemmata}

In this section we will set up some useful calculus results, which are easy to prove, but they are not easy to find in the literature (see 1] for more references). Hence we give full proofs. We point out that our compactness result is new.

2.1. The case when $I=R_{+}$. Assume that $u \in C_{0}^{1}\left(R_{+}\right)$with

$$
\int_{R_{+}}\left|u^{\prime}\right|^{2} d x \leq C^{2}
$$

where $C$ is a positive constant. Then, for any $x, y, \in R_{+}$, we have

$$
|u(x)-u(y)| \leq \int_{[x, y]}\left|u^{\prime}\right| d x .
$$

Using the Cauchy-Schwartz inequality we find

$$
|u(x)-u(y)| \leq C|x-y|^{1 / 2} .
$$

In particular, since $u(0)=0$, we have

$$
|u(x)| \leq C|x|^{1 / 2} .
$$


Given a positive function $p=p(x)$ on $R_{+}$satisfying

$$
\int_{R_{+}} p(x) x d x<+\infty
$$

we then have, for $R \geq 0$, that

$$
\int_{(R,+\infty)} p(x) u^{2} d x \leq C \int_{(R,+\infty)} p(x) x d x .
$$

Define $E$ as the completion of $C_{0}^{1}\left(R_{+}\right)$in the norm

$$
|u|=\left(\int_{R_{+}}\left|u^{\prime}\right|^{2} d x\right)^{1 / 2} .
$$

It is clear that the inequality $(*)$ is also true for every $u \in E$.

Let

$$
L^{2}\left(R_{+}, p d x\right)=\left\{u \in L_{l o c}^{1}\left(R_{+}\right) ; \int_{R_{+}} p u^{2}<+\infty\right\} .
$$

Then we have the following compactness result.

Proposition 2. The imbedding from $E$ to $L^{2}\left(R_{+}, p d x\right)$ is bounded and compact.

Proof. The boundedness of the imbedding is clear from the inequality $(*)$.

Now let $\left\{u_{k}\right\} \subset E$ with

$$
\int_{R_{+}}\left|u_{k}^{\prime}\right|^{2} d x \leq C^{2} .
$$

Then we have, for every $k$ and any $x, y \in R_{+}$,

$$
\left|u_{k}(x)-u_{k}(y)\right| \leq C|x-y|^{1 / 2} \text {. }
$$

In particular, take $y=0$ and we have

$$
\left|u_{k}(x)\right| \leq C|x|^{1 / 2} .
$$

Given $\epsilon>0$. By inequality $(*)$ we can choose $R>1$ sufficiently large such that for every $k$ we have

$$
\int_{[R,+\infty)} p u_{k}^{2} \leq C \int_{[R,+\infty)} x p(x) d x<\epsilon / 2 .
$$

By this and the Azela-Ascoli theorem we can find a subsequence, still denoted by $\left\{u_{k}\right\}$, such that it converges in $C_{l o c}^{0}\left(R_{+}\right)$, and hence in $L^{2}\left(R_{+}, p d x\right)$. This completes the proof of compactness part of the theorem.

Note that we cannot handle the case when $R_{+}$is replaced by the entire real line $R^{1}$ without further restriction. But we have another inequality, which will be introduced below. We remark that the inequality below cannot be used to prove a similar compactness result as above.

Using the compactness result, one readily gets the following result on the existence of the principal eigenvalue and function.

Theorem 3. Assume the function $f$ is positive somewhere on $R_{+}$, which is of the class $(P)$. Then there exist a positive function $V$ in $E$ and a positive number $\mu$ such that

$$
V^{\prime \prime}+\mu f(x) V=0, \quad \text { on } \quad R_{+} .
$$


2.2. The case when $I=R$. Assume that $p=p(x)$ is a positive function on the whole real line $R^{1}$. Now we define a Hilbert space $E^{\prime}$, which is the completion of the space $C_{0}^{1}\left(R^{1}\right)$ according to the Dirichlet integral. As before, we can define the space $L^{2}(R, p d x)$.

Definition. We say that a positive function $p$ satisfies the condition $(A B)$ if there are two positive constants $A$ and $B$ such that for any $x \in R$,

$$
p(x) \leq \frac{A}{(|x|+B)^{2}}
$$

Although the following result will not be used later in this work, we believe that it is a useful tool in the analysis of solutions of ordinary differential equations.

Proposition 4. Assume that the positive function $p$ on $R$ satisfies the condition $(A B)$. Then there is a positive constant $C$ such that for every $u \in E^{\prime}$, it holds that

$$
\int_{R} p(x) u^{2} d x \leq \frac{4 A}{B} u(0)^{2}+C \int_{R}\left|u^{\prime}\right|^{2} d x .
$$

We should mention that this inequality is a version of Hardy's inequality.

Proof. Without loss of generality, we may assume $A=1=B$ and take $u \in C_{0}^{1}(R)$. Note that

$$
\int_{R} \frac{1}{(|x|+1)^{2}} u^{2} d x=\int_{0}^{\infty} \frac{u^{2}}{(x+1)^{2}} d x++\int_{-\infty}^{0} \frac{u^{2}}{(x-1)^{2}} d x
$$

Then by integration by parts, we get

$$
\int_{0}^{\infty} \frac{u^{2}}{(x+1)^{2}} d x=u(0)^{2}+\int_{0}^{\infty} \frac{2 u u^{\prime}}{(x+1)} d x
$$

Similarly we have

$$
\int_{-\infty}^{0} \frac{u^{2}}{(x-1)^{2}} d x=u(0)^{2}+\int_{-\infty}^{0} \frac{2 u u^{\prime}}{(x-1)} d x
$$

Putting these two equalities together, we have

$$
\int_{R} \frac{1}{(|x|+1)^{2}} u^{2} d x=2 u(0)^{2}+\int_{R} \frac{2 \operatorname{sgn}(x) u u^{\prime}}{(|x|+1)} d x .
$$

Note that by the Cauchy-Schwartz inequality, we find

$$
\int_{R} \frac{2|u|\left|u^{\prime}\right|}{(|x|+1)} d x \leq \frac{1}{2} \int_{R} \frac{1}{(|x|+1)^{2}} u^{2} d x+8 \int_{R}\left|u^{\prime}\right|^{2} d x
$$

Hence we get

$$
\int_{R} \frac{1}{(|x|+1)^{2}} u^{2} d x=4 u(0)^{2}+16 \int_{R}\left|u^{\prime}\right|^{2} d x
$$




\section{AN APRIORI BOUND AND A COMPARISON LEMMA}

In this section, $a \in R$ is fixed. We write $M=R_{+}$.

As in the proof of Lemma 2.1 of [18] we have the following comparison lemma:

Lemma 5. Assume $\Omega$ is a bounded open subset in $M$. Suppose $u_{1}, u_{2} \in C^{2}(\Omega)$ are positive in $\Omega$, and they satisfy $T_{a}\left(u_{1}\right) \leq 0$ and $T_{a}\left(u_{2}\right) \geq 0$ on $\Omega$ and $u_{1} \geq u_{2}$ on $\partial \Omega$. Then $u_{1} \geq u_{2}$ on $\Omega$.

We now use this result to obtain the local existence of a boundary blow-up positive solution of $(1)_{a}$ on a bounded interval $G \subset M-M_{0}$. This result may be of independent interest (see [18]).

Proposition 6. Assume $a \in R$. There is at least one positive regular solution of the equation

$$
u^{\prime \prime}+a f(x) u-b(x) u^{p}=0 \quad \text { in } \quad G,
$$

with the boundary condition $u=+\infty$ on $\partial G$.

Proof. Fix an integer $k \in\{1,2,3, \ldots\}$. As in the introduction we let

$$
I(u)=\frac{1}{2} \int_{G}\left(\left|u^{\prime}\right|^{2}-a f(x) u^{2}\right)+\frac{1}{p+1} \int_{G} b(x)|u|^{p+1}
$$

be defined on

$$
E=\left\{u \in H^{1}(G) ;\left.u\right|_{\partial G}=k\right\} .
$$

It is easy to see that $I$ is coercive on $E$ (see section three for a more difficult case) and there is a minimizer, which is denoted by $u_{k}$, of $I$ on $E$.

By the comparison lemma above we have $u_{k} \leq u_{k+1}$, for $k=1,2, \ldots$. By a standard argument we can assume $u_{k} \rightarrow U_{a}$ in $C_{l o c}^{2}(G)$. This $U_{a}$ is what we wanted.

From the proof of the result above we can get the following:

Theorem 7. Assume that $M_{0}$ is non-empty and the set $M-M_{0}$ admits a bounded interval exhaustion, i.e., there is a bounded open subset sequence $\left\{\Omega_{j}\right\}$ such that $M-M_{0}=\bigcup \Omega_{j}$. Then for any $a \in R$, there is at least one positive smooth solution $\bar{U}_{a}$ of $T_{a}(u)=0$ on $M-M_{0}$ with the boundary blow-up condition $\bar{U}_{a}=+\infty$ on $M_{0}$.

We remark that when $M_{0}$ is empty and $a \leq 0$, there may be no positive solutions of $T_{a}(u)=0$ on $M$ at all (see [18]). One should also note that there is no contradiction between Theorem 1(iii) with our Theorem 5 because we require $T_{a}(u)=0$ on the whole $R_{+}$in Theorem 1 (iii).

\section{Existence AND Uniqueness of POSITIVE SOLUTions}

We will discuss the uniqueness of positive $E$-solutions of (1) $)_{a}$ for $a \in R$. The proofs of the following results are similar to the corresponding ones in [18, but for completeness, we give full proofs.

Lemma 8. For $a \in R$, there is at most one positive E-solution to (1)a.

Proof. Assume $u_{1}$ and $u_{2}$ are two positive $E$-solutions of $(1)_{a}$. We assume $u_{1} \neq u_{2}$, i.e., the set $M_{1}:=\left\{x \in M ; u_{1}(x) \neq u_{2}(x)\right\}$ has positive measure.

Let $\epsilon_{1}>\epsilon_{2}>0$ and denote

$$
v_{i}=\left(u_{i}+\epsilon_{i}\right)^{-1}\left[\left(u_{2}+\epsilon_{2}\right)^{2}-\left(u_{1}+\epsilon_{1}\right)^{2}\right]^{+}, \quad i=1,2,
$$


where [.] $]^{+}$is the positive part of [.]. Then each $v_{i}$ is an $H$-limit of a sequence in $H$ with compact support. Hence, we have for $i=1,2$,

$$
-\int\left[u_{i}^{\prime} v_{i}^{\prime}-a f u_{i} v_{i}\right]=\int b(x) u_{i}^{p} v_{i}
$$

By these we get

$$
-\int\left[u_{2}^{\prime} v_{2}^{\prime}-u_{1}^{\prime} v_{1}^{\prime}\right]=\int a f(x)\left[u_{1} v_{1}-u_{2} v_{2}\right]+\int b(x)\left(u_{2}^{p} v_{2}-u_{1}^{p} v_{1}\right] .
$$

Note that the left side is

$$
-\int\left(\left|u_{2}^{\prime}-\frac{u_{2}+\epsilon_{2}}{u_{1}+\epsilon_{1}} u_{1}^{\prime}\right|^{2}+\left|u_{1}^{\prime}-\frac{u_{1}+\epsilon_{1}}{u_{2}+\epsilon_{2}} u_{2}^{\prime}\right|^{2}\right)
$$

which is non-positive and goes to

$$
-\int\left(\left|u_{2}^{\prime}-\frac{u_{2}}{u_{1}} u_{1}^{\prime}\right|^{2}+\left|u_{1}^{\prime}-\frac{u_{1}}{u_{2}} u_{2}^{\prime}\right|^{2}\right) \leq 0 .
$$

The first term on the right goes to zero as $\epsilon_{1} \rightarrow 0$. The second term on the right goes to

$$
\int b(x)\left(u_{2}^{p-1}-u_{1}^{p-1}\right)\left(u_{2}^{2}-u_{1}^{2}\right)
$$

which is non-negative by our assumption. Hence, $M_{1} \subset M_{0}$. By all these we get that

$$
\int_{M_{0}}\left(\left|u_{2}^{\prime}-\frac{u_{2}}{u_{1}} u_{1}^{\prime}\right|^{2}+\left|u_{1}^{\prime}-\frac{u_{1}}{u_{2}} u_{2}^{\prime}\right|^{2}\right)=0
$$

and

$$
u_{2}=c u_{1}, \quad \text { on } \quad M_{0}
$$

for some constant $c \neq 1$, a contradiction.

In the rest of this section we assume that $a \in\left(\lambda_{1}, \lambda_{*}\right)$. We now prove part (i) of Theorem 1:

Proof. Recall that we assume $M_{0}$ is bounded. It is clear that the functional $J$ is weakly lower semi-continuous on $E$ according to our compactness result. We claim that

$$
J(u) \rightarrow+\infty \quad \text { as } \quad\|u\| \rightarrow+\infty .
$$

Assume not. Then we have the sequence $\left\{u_{n}\right\} \subset H$ such that $J\left(u_{n}\right)$ is bounded above and $\left\|u_{n}\right\| \rightarrow \infty$. Then we have that $d_{n}^{2}:=\int f(x) u_{n}^{2} \rightarrow \infty$; for otherwise, $\left\{\left\|u_{n}\right\|\right\}$ is bounded.

Set

$$
\bar{u}_{n}=u_{n} / d_{n} .
$$

Then we have $\int f(x) \bar{u}_{n}^{2}=1$ and

$$
\limsup \int_{M}\left|\bar{u}_{n}^{\prime}\right|^{2}-a+\frac{2}{(p+1)\left\|u_{n}\right\|_{2}^{2}} \int_{M} b u_{n}^{p+1} \leq 0 .
$$


By this, we may assume that $\left\|\bar{u}_{n}\right\|+\int_{M} b u_{n}^{p+1}$ is uniformly bounded. Hence, we may assume further that $\bar{u}_{n} \rightarrow \bar{u}$ weakly in $E$, almost everywhere, and strongly in $L^{2}\left(R_{+}, p\right)$. Then by Fatou's lemma, we get

$$
\int_{R_{+}} b(x) \bar{u}^{p+1}=0 .
$$

Hence, $\bar{u}=0$ on the set $R_{+}-M_{0}$. Therefore, we can use the strong convergence on $L^{2}\left(R_{+}, p\right)$ to get $\int_{M_{0}} f(x) \bar{u}^{2}=1$ and

$$
\int_{M_{0}}\left|\bar{u}^{\prime}\right|^{2} \leq a
$$

By this and the definition of $\lambda_{*}$ we have

$$
\lambda_{*} \leq a .
$$

This is a contradiction with our hypothesis at the beginning.

Therefore, we have a minimizer $u_{0}$, which is a non-negative function, of the functional $J$ on $E$. To prove that $u_{0}$ is non-trivial, we take a large interval $I_{R}$ with $M_{0} \subset I_{R}$ such that the first eigenvalue $\lambda_{1}\left(I_{R}\right)-a \leq-\epsilon$ for some small $\epsilon>0$. Let $\phi_{1}$ be the eigenfunction of the eigenvalue $\lambda_{1}\left(I_{R}\right)$ of the weighted quotient $Q\left(u, I_{R}\right)$ and $\int_{I_{R}} f(x) \phi^{2}=1$. Then, for small $t>0$,

$$
J\left(t \phi_{1}\right)=\frac{\lambda_{1}\left(I_{R}\right)-a}{2} t^{2}+\frac{t^{p+1}}{p+1} \int_{I_{R}} b \phi^{p+1}<0 .
$$

Note that $J(0)=0$. Hence $u_{0}$ is non-trivial and $u_{0}>0$ everywhere on $R_{+}$by the strong maximum principle. The smoothness of $u_{0}$ follows from the standard argument.

For the uniqueness of positive solutions, we use Lemma 8 above. Hence the proof is complete.

Remark. By uniqueness of positive solutions and the barrier method, we know immediately that, for $x \in R_{+}, a \rightarrow u_{a}(x)$ is increasing (for a proof, one may see [18]). This fact will be used later.

\section{Blow-UP OF the SOlution SEQUence}

In this section we assume $\lambda_{*}<+\infty$.

We study the behavior of the solution sequence when $a_{n} \rightarrow \lambda_{*}^{-}$. We assume $a_{n}>\lambda_{1}$. Set $u_{n}=u_{a_{n}}$.

We now prove Theorem 1(ii). First we note that

$$
\lim _{n \rightarrow+\infty} \int_{R_{+}} f(x) u_{n}^{2}=+\infty .
$$

Otherwise, we can find a minimizer, which is the limit of $u_{n}$, of $J$ as in Theorem $1(\mathrm{i})$, and this is impossible.

As in the proof of Theorem 1(i), we set

$$
\bar{u}_{n}=u_{n} / d_{n} .
$$

Then we find $\bar{u} \in H$ with $\bar{u}=0$ on $R_{+}-M_{0}$. Note that

$$
\bar{u}_{n}^{\prime \prime}+a_{n} f(x) \bar{u}_{n}=0
$$

on $M_{0}$. We can use the standard estimate [10] to conclude the uniform convergence of $\bar{u}_{n}$ to $\bar{u}$ on $K$. This implies that $u_{n}(x) \rightarrow+\infty$ on $K$. 


\section{NON-EXISTENCE OF POSITIVE SOLUTIONS}

We prove in this section the third part of Theorem 1. That is, under the assumption $\lambda_{*}<+\infty$, there is no positive solution of $(1)_{a}$ when $a \geq \lambda_{*}$.

Assume that $a=\lambda_{*}$ and there is a positive solution, say $u$, of (1)a. Let $v$ be the $\lambda_{*}$-eigenfunction on $M_{0}$ which satisfies the equation

$$
u^{\prime \prime}+a f(x) u=0 \quad \text { on } \quad M_{0}
$$

and the boundary condition $v=0$ on $\partial M_{0}$. Set $\phi=v / u$. Then by direct computation we find

$$
\phi^{\prime \prime}+2 \phi^{\prime}(\log u)^{\prime}=0 \quad \text { on } \quad M_{0}
$$

and $\phi=0$ on $\partial M_{0}$. However, this gives an absurd result that $\phi=0$ on $M_{0}$ by the standard maximum principle (see Theorem 3.1 in [10]). Therefore, there is no positive solution in this case.

In the case where $a>\lambda_{*}$, if there is a positive $E$-solution of $(1)_{a}$, say $u_{*}$, then using the barrier method and the uniqueness result we find that $u_{a}(x) \leq u_{*}(x)$ for all $x \in M$ and $a<\lambda_{*}$. Therefore, there is no blow-up on $M_{0}$ for any sequence of $\left(u_{a}\right)$, which contradicts Theorem 1(ii). Hence, we get the conclusion of Theorem 1 (iii).

We can give another proof for positive $E$-solutions when $a>\lambda\left(M_{0}\right)$. Take a smooth bounded domain $G$ inside $M_{0}$ such that $\lambda_{1}(G)<a$. Let $w_{1}$ be the positive eigenfunction associated to $\lambda_{1}(G)$. Therefore, we can find a function $u_{0} \in E$ such that the number

$$
\mu=\int_{G}\left(u_{0}^{\prime} w_{1}^{\prime}-a f(x) u_{0} w_{1}\right)
$$

is positive (see also [3]). Otherwise, we have

$$
\int_{R_{+}}\left(u^{\prime} w_{1}^{\prime}-a f(x) u w_{1}\right) \leq 0
$$

for every $u \in H$. Hence, using $-u$ as a test function, we have

$$
\int_{R_{+}}\left(u^{\prime} w_{1}^{\prime}-a f(x) u w_{1}\right)=0
$$

for every $u \in H$. Therefore,

$$
-w_{1}^{\prime \prime}-a f(x) w_{1}=0 \quad \text { on } \quad R_{+} .
$$

It is absurd with the definition of $w_{1}$.

Let

$$
u_{t}=t w_{1}+u_{0} .
$$

Then we compute $J\left(u_{t}\right)$ and find

$$
J\left(u_{t}\right)=\left(\lambda_{1}(G)-a\right) t^{2} / 2+\mu t+d
$$

where

$$
d=\frac{1}{p+1} \int_{R_{+}} b u_{0}^{p+1}+\frac{1}{2} \int_{R_{+}}\left(\left|u_{0}^{\prime}\right|^{2}-a f(x) u_{0}^{2}\right) .
$$

Hence, $J\left(u_{t}\right) \rightarrow-\infty$ as $t \rightarrow-\infty$, which contradicts the coercivity of $J$. So we conclude the result when $a>\lambda_{*}$. 


\section{The REAL LINE CASE}

In this section we discuss the equations $(1)_{a}$ on the real line $R$. We point out that the method used here cannot work for the case when $b(x)=0$ somewhere.

We assume that $f$ is a bounded integrable function on $R$, and $b(x)$ is positive and uniformly bounded away from zero, that is, there is a uniform constant $c>0$ such that

$$
b(x) \geq c>0 \quad \text { for } \quad x \in R .
$$

For example, $b(x)=2+\sin (x)$ satisfies condition $(e)$.

We assume $a \in\left(\lambda_{1}, \lambda_{*}\right)$.

Set $I_{k}=(-k, k)$ for $k=1,2, \ldots$. Then as before, we define the functional

$$
J_{k}(u)=\frac{1}{2} \int_{I_{k}}\left(\left|u^{\prime}\right|^{2}-a f(x) u^{2}\right)+\frac{1}{p+1} \int_{I_{k}} b(x)|u|^{p+1}
$$

on $E_{k}:=H_{0}^{1}\left(I_{k}\right)$. Clearly, the functional is bounded below (see [1]) on $E_{k}$. We can easily get a minimizer $u_{k} \in E_{k}$ such that

$$
J_{k}\left(u_{k}\right)=i n f_{u \in E_{k}} J_{k}(u),
$$

and therefore we get a family of positive point-wise increasing functions $\left(u_{k}\right)$, which satisfy the equations $(1)_{a}$ with the Dirichlet boundary condition $u_{k}(k)=0=$ $u_{k}(-k)$.

Using the maximum principle we can easily show that $u_{k}$ is uniformly bounded on $R$. In fact, at the maximum point $x_{0} \in I_{k}$, we have that $u^{\prime \prime}\left(x_{0}\right) \leq 0$. Hence, by using our equation $(1)_{a}$ we find

$$
a f\left(x_{0}\right) u\left(x_{0}\right)=b\left(x_{0}\right) u\left(x_{0}\right)^{p}-u^{\prime \prime}\left(x_{0}\right) \geq c u\left(x_{0}\right)^{p}
$$

and $u\left(x_{0}\right) \leq[a D / c]^{1 /(p-1)}$, where $D=\sup _{x \in R} f_{+}(x)$. According to our assumption on $p$, we know that $u_{k}$ is uniformly bounded in $L^{2}(R, p d x)$.

Choose $\xi \in C_{0}^{1}\left(I_{1}\right)$ and we have

$$
J_{k}\left(u_{k}\right) \leq J_{1}(\xi)
$$

for every $k=1,2, \ldots$. Using this bound and the uniform boundedness of the sequence $\left(u_{k}\right)$ we obtain a uniform bound of $\int_{R}\left|u_{k}^{\prime}\right|^{2} d x$. In fact, multiplying both sides of equation (1) for $u_{k}$ by $u_{k}$ and integrating by parts, we know that the integrals

$$
\int_{R} u_{k}^{p+1} d x+\int_{R}\left|u_{k}^{\prime}\right|^{2} d x
$$

are uniformly bounded. Therefore, we can pass to a subsequence, still denoted by $\left(u_{k}\right)$, which converges in $C_{l o c}^{2}(R)$ to a positive solution $U$ of $(1)_{a}$ on $R$. It is easy to see that

$$
\int_{R}\left|U^{\prime}\right|^{2} d x+\int_{R} U^{p+1} d x<+\infty
$$

and $U$ is in $L^{2}(R, p d x)$. Hence by a standard estimate (see [18]), we have proven that this $U$ is in $E^{\prime}$. That is to say, $U(x) \rightarrow 0$ uniformly as $|x| \rightarrow \infty$. As before, we can prove the uniqueness of the $E^{\prime}$-solution. 
We point out that for the case when $a \in\left(0, \lambda_{1}\right]$, we can easily show by the maximum principle that there is no positive $E^{\prime}$-solution of $(1)_{a}$.

In summary, we have

Theorem 9. Assume $f$ is a bounded, positive somewhere, integrable function on $R$. Assume that the positive function $b(x)$ satisfies $(e)$ above. Then

(i) for $a \in\left(0, \lambda_{1}\right]$ there is no positive $E^{\prime}$-solution of $(1)_{a}$;

(ii) for any $a \in\left(\lambda_{1},+\infty\right)$ there is a positive $E^{\prime}$-solution of $(1)_{a}$, and this solution is unique in $E^{\prime}$.

We remark that some extension of Theorem 9 to higher dimensions has been obtained by Du and Ma 18].

\section{REFERENCES}

1. G.Buttazzo, M.Giaquinta, and S.Hildebrandt, One-dimensional variational problems, Oxford scientific publishing, Clarendon press, Oxford, 1998. MR 2001a:49001

2. Z.R.Jin, Principal eigenvalues with indefinite weight functions, Trans. AMS, 349(1997)19451959. MR 97h:35056

3. J.Lopez-Gomez and J.C.Sabina de Lis, First variation of principal eigenvalues, J.Diff. Equa., 148(1998)47-64. MR 99f:35033

4. K.J.Brown, C.Cosner, and J.Fleckinger, Principal eigenvalues for problems with indefinite weight function on $R^{N}$, Proc.AMS, 109(1990)147-155. MR 90m:35140

5. W.Allegretto, Principal eigenvalues for indefinite weight elliptic problems in $R^{N}$, Proc. AMS, 116(1992)701-706. MR 93a:35114

6. J.Kazdan and F.Warner, Scalar curvature and conformal deformation of Riemannian structure, J.Diff. Geom., 10(1975)113-134. MR 51:1661

7. T.Ouyang, On the positive solutions of semilinear equation $\triangle u+\lambda u-h u^{p}=0$ on the compact manifolds, Trans. AMS, 331(1992)503-527. MR 92h:35012

8. M.A. del Pino, Positive solutions of a semilinear elliptic equations on a compact manifold, Nonlinear Analysis, 22(1994)1423-1430. MR 95g:58256

9. A.Edelson and A.Rumbos, Linear and semilinear eigenvalue problems in $R^{n}$, Comm. Partial Diff. Equations, 18, 215-240(1993). MR 94b:35101

10. Y.Du, Q.Huang, Blow-up solutions for a class of semilinear elliptic and parabolic equations, SIAM J.Math. Anal., 31(1999)1-18. MR 2000g:35059

11. L. Ma, Conformal deformations on a noncompact riemannian manifold, Math. Ann., 295(1993)75-80. MR 93k:53040

12. P.Li, L.F.Tam, D.Yang, On the elliptic equation $\triangle u+k u-K u^{p}=0$ on complete Riemannian manifolds and their geometric applications, Trans. AMS, 350(1998)1045-1078. MR 98e:58175

13. W.M.Ni, On the elliptic equation $\triangle u+K(x) u^{(n+2) /(n-2)}=0$, its generalizations, and applications in geometry, Indiana Univ. Math. J., 31(1982)495-529. MR 84e:35049

14. P.Aviles, R.McOwen, Conformal deformation to constant negative scalar curvature on noncompact Riemannian manifolds, J.Diff.Geom., 27(1988)225-239. MR 89b:58225

15. D.Gilbarg and N.S.Trudinger, Elliptic Partial Differential Equation of Second Order, Springer-Verlag, 1977. MR 57:13109

16. F.H.Lin, On the elliptic equations $D_{i}\left[A_{i j} D_{j} U\right]-k(x) U+K(x) U^{p}=0$, Proc. AMS, 95(1985)219-226. MR 86k:35041

17. M.Marcus and L.Veron, Uniqueness and asymptotic behavior of solutions with boundary blow-up for a class of nonlinear elliptic equations, Anal. Nonlinéaire, 14(1997)237-274. MR 97m:35068

18. Y.H.Du and L. Ma, Positive solutions of an elliptic partial differential equation on $R^{n}$, preprint, 2000.

19. Y.H.Du and L.Ma, Logistic type equations on $R^{n}$ by a squeezing method involving boundary blow-up solutions, Journal of London Mathematical Society, 64(2001)107-124. 
20. G.A.Afrouzi and K.J.Brown, On a diffusive logistic equation, JMAA, 225(1998)326-339. MR 99g:35130

21. E.N.Dancer and S.P.Hasting, On the global bifurcation diagram for the one dimensional Ginzburg-Landau model of superconductivity, European J. Applied Math., 11(2000)271-291. CMP 2001:16

Department of Mathematical Sciences, Tsinghua University, Beijing 100084, People's Republic of China

E-mail address: lma@math.tsinghua.edu.cn

Department of Mathematics, The National University of Singapore, 10 Kent Ridge Crescent, Singapore 119260

E-mail address: matxuxw@math.nus.edu.sg 